\title{
All-oral triplet combination of ixazomib, lenalidomide, and dexamethasone in newly diagnosed transplant- eligible multiple myeloma patients: final results of the phase II IFM 2013-06 study
}

High-dose therapy with autologous stem cell transplantation (ASCT) is considered as a standard of care for patients with transplant-eligible symptomatic newly-diagnosed multiple myeloma (NDMM). ${ }^{1}$ The benefit of ASCT in such patients has been recently confirmed by two phase III randomized trials demonstrating better progression-free survival and/or overall survival in the transplant arm..$^{2-4}$ In the past decades, induction therapy before ASCT has been improved dramatically, resulting in deeper responses and prolonged progression-free survival. The triplet combination of bortezomib, lenalidomide and dexamethasone (VRD) is one of the standard-of-care induction regimens in the context of transplantation. .,2, $^{1 \times a-}$ zomib is the first-in-class oral proteasome inhibitor approved for patients with relapsed/refractory multiple myeloma in combination with lenalidomide and dexamethasone. ${ }^{6}$ Here, we report the results of the multicenter, open-label, phase II study by the Intergroupe Francophone du Myelome (IFM), 2013-06, evaluating the efficacy and safety of ixazomib, lenalidomide and dexamethasone (IRD) used as an induction and consolidation regimen followed by ixazomib maintenance in transplanteligible patients with NDMM.

This study included transplant-eligible patients with previously untreated symptomatic NDMM. Key selection criteria are indicated in Online Supplementary Figure S1. All patients provided written informed consent to participation in the study, which was approved by relevant national health authorities and ethics committees and was conducted in accordance with the International Conference on Harmonization of Good Clinical Practice guidelines and the principles of the Declaration of Helsinki. This clinical trial is registered at www.clinicaltrials.gov as NCT01936532 and with EUDRACT number 2013-001443-3. Induction therapy comprised three 28-day cycles of oral ixazomib (4 mg on days 1, 8, and 15), oral lenalidomide (25 $\mathrm{mg}$ on days 1-21) and oral dexamethasone (40 mg on days $1,8,15$, and 22). Stem cell harvest was planned for all patients after high-dose cyclophosphamide $\left(3 \mathrm{~g} / \mathrm{m}^{2}\right)$ plus granulocyte colony-stimulating factor). Patients proceeded to transplant using melphalan $200 \mathrm{mg} / \mathrm{m}^{2}$ as the conditioning regimen. Patients whose disease did not progress then proceeded to early consolidation therapy with two 28-day cycles of IRD, followed by late consoli- dation with six 28-day cycles of ixazomib (4 mg on days 1 , 8 , and 15 ) and lenalidomide (25 mg on days 1-21) without dexamethasone. Patients subsequently received maintenance therapy with ixazomib (4 $\mathrm{mg} /$ day on days 1,8 , and 15) for 1 year.

The primary endpoint was stringent complete response (SCR) rate at the completion of extended consolidation. Secondary endpoints included response at each step of the program, time to response, quality of stem cell harvest, progression-free survival, overall survival, and safety. Myeloma response assessment was based on the International Myeloma Working Group uniform response criteria. ${ }^{7}$ SCR was defined as complete response (CR) with the addition of normal serum free light chain ratio and absence of clonal plasma cells in the bone marrow, as assessed by flow cytometry analysis. All patients were followed until death or end of the study (June 2020).

Forty-two eligible patients were enrolled between November 2014 and May 2015. The patients' characteristics are summarized in Table 1. Their median age was 60 years. Eight $(19 \%)$ patients had a high-risk cytogenetic profile.

Table 1. Patients' demographic and baseline disease characteristics.

\begin{tabular}{|l|c|}
\hline Characteristic & $\mathbf{N = 4 2}$ \\
\hline Gender: male/female, N & $21 / 21$ \\
\hline Median age, years (range) & $60(43-66)$ \\
\hline $\begin{array}{l}\text { ECOG PS, N (\%) } \\
0,1,2\end{array}$ & $23(55), 15(36), 4(9)$ \\
\hline $\begin{array}{l}\text { Isotype, N (\%) } \\
\text { IgG, IgA, light chain only }\end{array}$ & $27(64), 9(22), 6(14)$ \\
\hline $\begin{array}{l}\text { ISS stage, N (\%) } \\
\text { I, II, III }\end{array}$ & $12(29), 23(55), 7(17)$ \\
\hline $\begin{array}{l}\text { Median creatinine, } \mu \text { mol/L, (range) } \\
\text { Cytogenetic risk profile, N (\%) } \\
\text { High-risk }\end{array}$ & $72(48-134)$ \\
\hline $\begin{array}{l}\text { Standard } \\
\text { Stem-cell collection } \\
\text { Median CD34 cell yield (x 10 } / \mathrm{kg})\end{array}$ & $8(19)$ \\
\hline
\end{tabular}

*High-risk cytogenetics was defined by the presence of $t(4 ; 14)$ (with a positive cut-off at $30 \%$ ) and/or 17p deletion (with a positive cut-off at 50\%). ECOG: Eastern Cooperative Oncology Group; PS: performance status; Ig: immunoglobulin; ISS: International Staging System. 
The patients' disposition in the study program is summarized in Online Supplementary Figure S1. Overall, 40 (95\%) patients completed induction and 37 (88\%) underwent ASCT. Stem cell collection failed in one patient. Plerixafor was needed for stem cell mobilization in five patients.

By the end of induction $(n=42)$, the overall response rate was $80 \%(n=33)$, including $30 \%(n=10)$ very good partial responses and $12 \%(n=5) C R / s C R$ (Figure $1 A)$. At the end of consolidation (primary endpoint) $(n=37)$, the SCR rate was $41 \%$ (33\% in an intention-to-treat analysis). The median time to partial response and CR was 1 and 8 months, respectively. As of June 2020, the median follow-up from the start of therapy was 62.6 months. Twenty-nine patients had progressive disease and seven patients died due to myeloma progression. The median progression-free survival was 41.8 months (95\% confidence interval: $33.2-$ 62 ) and the 3-year overall survival was $92.8 \%$ (95\% confidence interval: 85.3-100) (Figure 1B, C).

There were no IRD-related deaths. Overall, seven (16.6\%) patients discontinued treatment permanently due to treatment-related toxicity: one patient during induction (skin rash), three during consolidation (1 skin rash, 2 thrombocytopenia) and three patients during maintenance (colon cancer, thrombocytopenia, pneumonia). For these patients, the median time to ixazomib discontinuation was 227 days. Overall, 33 (79\%) patients had at least one dose modification of one of the study drugs. A dose reduction of ixazomib, lenalidomide or dexamethasone occured in $60 \%, 67 \%$ and $29 \%$ of patients, respectively. Adverse events reported for at least $10 \%$ of patients are described in Table 2. During induction, grade 3-4 neutropenia was the most frequent treatment-related adverse event, occurring in eight (19\%) patients. Skin rash was reported in 12 (29\%), including 5\% with grade 3-4. During consolidation, neutropenia and thrombocytopenia were the most frequent adverse events, with grade 3-4 events occuring in 14 (38\%) and eight (22\%) patients, respectively. During maintenance, thrombocytopenia and lung infection were the most frequent adverse events, occurring in ten (32\%) and 12 (39\%) patients, respectively. Grade 1-2 sensory peripheral neuropathy was reported in 12 (29\%) patients, including two with grade 2 peripheral neuropathy. Deep-vein thrombosis occurred in one patient.

The primary objective of this phase II study was to evaluate the efficacy of a transplant program with the oral triplet IRD as induction and consolidation in NDMM patients. In the intention-to-treat population $(n=42)$, the overall response rate was $92.3 \%$, including $70.3 \%$ with a very good partial response or better. Our study showed that responses continuously deepened throughout the program. At the completion of extended consolidation, the per protocol $\mathrm{CR} / \mathrm{SCR}$ rate was $44 \%$ (37\% in intention-to-treat analysis). These response rates are close to those obtained with VRD as the induction/consolidation regimen in the IFM-2009 and GEM2012 trials. $^{2,5}$ However, patients
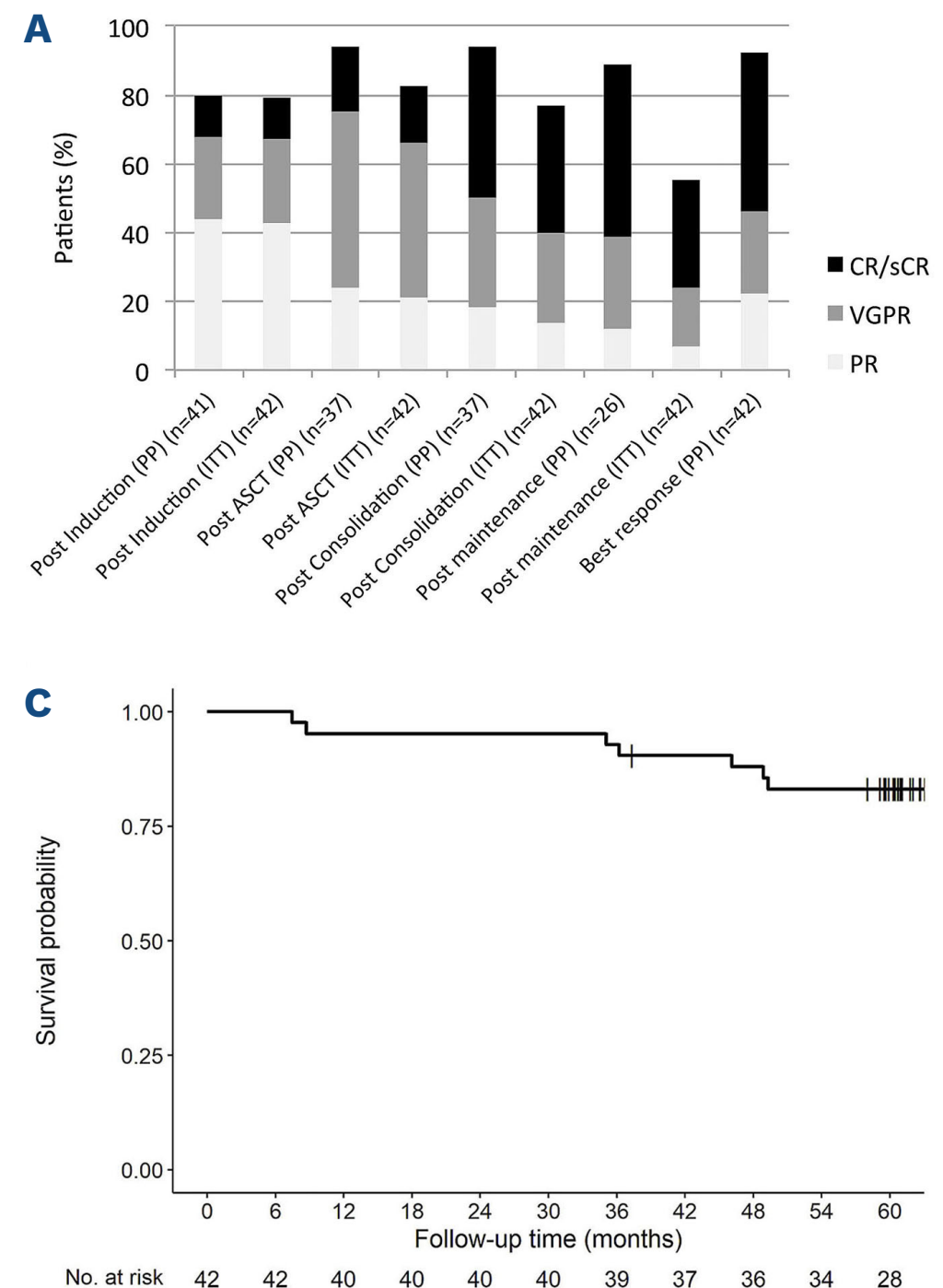

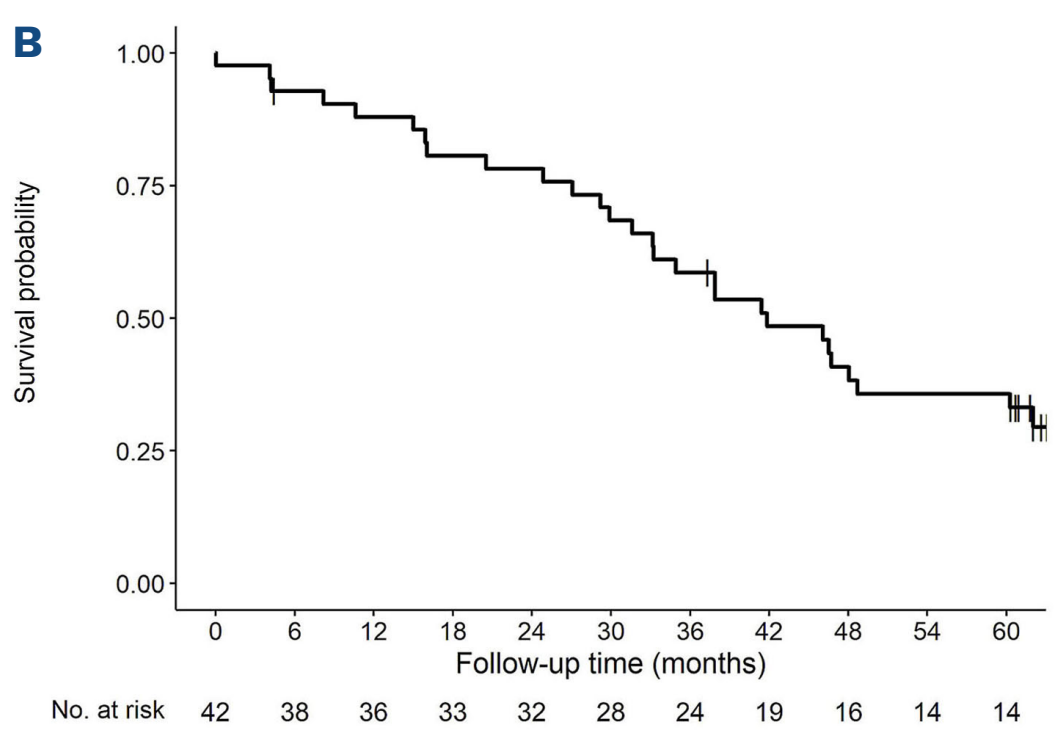

Figure 1. Efficacy of the all-oral ixazomib, lenalidomide, and dexamethasone transplant program. (A) Response rate. (B) Progression-free survival. (C) Overall survival. The median duration of the follow-up was estimated using the reverse Kaplan-Meier method. Progression-free survival was calculated as the time from the start of treatment to the first documentation of progressive disease, or death if the patient died due to any cause before progression. Overall survival was calculated as the time from the start of treatment to death. The Kaplan-Meier method was used to estimate the survival distribution. All analyses were conducted using $\mathrm{R}$ version 4.0. ASCT: autologous stem cell transplantation; CR: complete remisison; sCR: stringent complete remission; VGPR: very good partial response; PR: partial response. ITT: intention-to-treat; PP: per protocol. 
Table 2. Adverse events reported through induction, consolidation and maintenance.

Induction $(\mathrm{N}=42)$

\begin{tabular}{|c|c|c|}
\hline Adverse event & $\begin{array}{c}\text { Any grade } \\
\text { Patients (\%) }\end{array}$ & $\begin{array}{c}\text { Grade 3/4 } \\
\text { Patients (\%) }\end{array}$ \\
\hline
\end{tabular}

\section{Hematologic}

Neutropenia

Thrombocytopenia

$8(19)$

$1(2)$

8 (19)

0

\section{Non-hematologic}

\begin{tabular}{l|l}
\hline Constipation & $8(19)$ \\
\hline Diarrhea & $8(19)$ \\
\hline
\end{tabular}

\begin{tabular}{l|c}
\hline Nausea & $10(24)$ \\
\hline Pneumonia/bronchitis & $8(19)$ \\
\hline
\end{tabular}

Skin rash $12(29)$

6 (13)

\begin{tabular}{c|c}
\hline$(19)$ & 0 \\
\hline $8(19)$ & 0 \\
\hline $0(24)$ & 0 \\
\hline$(19)$ & $5(12)$ \\
\hline $2(29)$ & $2(5)$ \\
$6(13)$ & 0 \\
\hline
\end{tabular}

0

\begin{tabular}{l|l}
0 & $7(19)$
\end{tabular}

6 (16)

$6(16)$

10 (27)

8(22)

Maintenance $(\mathrm{N}=31)$

\begin{tabular}{|c|c|c|}
\hline $\begin{array}{c}\text { Grade } 3 / 4 \\
\text { Patients (\%) }\end{array}$ & $\begin{array}{c}\text { Any grade } \\
\text { Patients (\%) }\end{array}$ & $\begin{array}{c}\text { Grade } 3 / 4 \\
\text { Patients (\%) }\end{array}$ \\
\hline
\end{tabular}

Patients (\%)

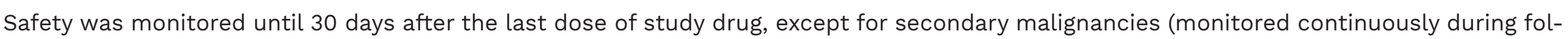

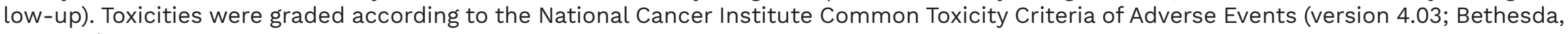
MD, USA).

in the present study received a higher number of cycles (induction, $n=3$; early consolidation, $n=2$; late consolidation, $n=6$ ) in comparison with patients from IFM-2009 (5 cycles of VRD) or GEM2012 (8 cycles of VRD). In the present study, patients received ixazomib maintenance for 1 year, with no significant improvement in CR/SCR rates. The phase III, placebo-controlled TOURMALINE-MM3 trial demonstrated a modest progression-free survival benefit in NDMM patients receiving post-ASCT ixazomib maintenance for 2 years (26.5 vs. 21.3 months at the start of maintenance). ${ }^{8}$ After a median follow-up of nearly 5 years, the median progression-free survival observed in the present study was 41.8 months with a 3-year overall survival of 92.8\%. Continuous ixazomib therapy following ASCT in combination with lenalidomide and dexamethasone maintenance has been evaluated (and compared with lenalidomide and dexamethasone) in the randomized phase III trial GEM2014 ( $n=332)$. After a median follow-up of 56 months, the addition of ixazomib did not result in a progression-free survival benefit. ${ }^{9}$ At the time the study was designed, continuous lenalidomide maintenance after ASCT did not demonstrate a benefit on overall survival and was not appoved. ${ }^{10}$ In the present study, a fixed-duration maintenance with ixazomib alone appears to be a suboptimal approach for transplant-eligible NDMM patients.

Safety was an important objective of the present phase II trial. The strategy was feasible, with seven (16.6\%) patients discontinuing therapy due to treatment toxicity and no IRD-related mortality. Overall, 33 (79\%) patients had at least one dose modification of one study drug. The hematologic toxicities were predictable and manageable.
The most common hematologic toxicity related to IRD was thrombocytopenia with grade >3 occuring in 29 (69\%) patients. Thrombocytopenia related to the IRD combination was expected and has been described previously. ${ }^{6,8,11}$ Considering non-hematologic toxicities, skin rash occurred in 23 (54\%) patients, with only two (5\%) grade 3/4 adverse events. Grade $\geq 2$ peripheral neuropathy occurred in two patients and one patient had grade $\geq 3$. These results compare favorably with those of VRD strategies with a rate of grade $>3$ peripheral neuropathy of $12 \%$ and $4 \%$ in the IFM-2009 and GEM2012 studies, respectively. ${ }^{2,5}$ The triplet combination of carfilzomib, lenalidomide and dexamethasone (KRD) with transplantation demonstrated strong efficacy results but is associated with substantial cardiac events. ${ }^{12}$ In the present study, no patient developed treatment-related cardiac failure.

To conclude, a transplant program with all-oral IRD as induction and consolidation, followed by 1 year of maintenance with ixazomib is effective in NDMM patients and has a favorable safety profile. However, these results are inferior, with respect to progression-free survival, to those achieved with VRD ASCT and lenalidomide maintenance. To date, ixazomib-based combinations have failed to significantly improve the outcome of transplant-eligible patients with NDMM.8,9 This suboptimal efficacy can be partially explained by inferior in vitro proteasome inhibition with ixazomib in comparison with other proteasome inhibitors. ${ }^{13}$ In NDMM patients, ixazomib could however be suitable for a specific subset of frail patients with comorbidities (e.g., pre-existing neuropathy, cardiac insufficiency), thought not to be able to tolerate bortezomib or carfilzomib-based combinations. Recently, daratu- 
mumab in combination with bortezomib, thalidomide and dexamethasone was approved for transplant-eligible NDMM patients and is now considered as a standard of care. ${ }^{1.14}$ The phase II randomized study GRIFFIN also demonstrated strong efficacy results (without a safety signal) with daratumumab in combination with VRD in transplant-eligible NDMM patients. ${ }^{15}$ One way of improving IRD efficacy in the context of transplantation could be the addition of anti-CD38 antibody. The convenience and efficacy profile of IRD in transplant-eligible NDMM patients led to the design of the IFM phase II study 2018-01 (NCT03669445) to evaluate the efficacy and safety of IRD in combination with daratumumab in such patients. Based on their efficacy/safety profiles, bortezomib or carfilzomib-based induction regimens with anti-CD38 should be considered as a standard of care for transplant-eligible NDMM patients.

\section{Authors}

Cyrille Touzeau, ${ }^{1,2,3}$ Aurore Perrot, ${ }^{4}$ Murielle Roussel, ${ }^{5}$ Lionel Karlin, ${ }^{6}$ Lotfi Benboubker, ${ }^{7}$ Caroline Jacquet, ${ }^{8}$ Mohamad Mohty, ${ }^{9}$ Thierry Facon, ${ }^{10}$ Salomon Manier, ${ }^{10}$ Marie-Lorraine Chretien, ${ }^{11}$ Mourad Tiab, ${ }^{12}$ Cyrille Hulin, ${ }^{13}$ Xavier Leleu, ${ }^{14}$ Hervé Avet Loiseau, ${ }^{4}$ Thomas Dejoie, ${ }^{15}$ Lucie Planche, ${ }^{16}$ Michel Attal $^{4}$ and Philippe Moreau ${ }^{1,2,3}$

1Service d'Hématologie, Centre Hospitalier Universitaire (CHU) Hotel Dieu, Nantes; ${ }^{2}$ CRCINA, INSERM, CNRS, Université d'Angers, Université de Nantes, Nantes; ${ }^{3}$ Site de Recherche Intégrée sur le Cancer (SIRIC) «ILIAD», INCA-DGOS-INSERM 12558, Nantes; ${ }^{4} \mathrm{CHU}$ de Toulouse, IUCTO, Université de Toulouse, UPS, Service d'Hématologie, Toulouse; ${ }^{5}$ Hématologie Clinique et Thérapie Cellulaire, CHU Limoges; ${ }^{6} \mathrm{Hôpital}$ Lyon Sud, Pierre-Benite; ${ }^{7}$ Service Hématologie et Thérapies Cellulaires, CHRU Bretonneau, Tours; ${ }^{8}$ Service d'Hématologie, $\mathrm{CHU}$ Nancy, Vandoeuvre-lès-Nancy; ${ }^{9}$ Hématologie Clinique et Thérapie Cellulaire, Hôpital Saint Antoine, Sorbonne Université, INSERM UMRS 938, Paris; ${ }^{10}$ Maladies du Sang, CHRU de Lille, Lille; ${ }^{11}$ Hématologie Clinique, CHU Dijon Bourgogne, Dijon; ${ }^{12}$ Service d'Hématologie, Centre Hospitalier Departemental, La Roche sur Yon; ${ }^{13}$ Service d'Hématologie, Hôpital Haut-Lévêque, CHU de Bordeaux, Pessac; ${ }^{14}$ Service d'hématologie, $\mathrm{CHU}$ de Poitiers, Portiers; ${ }^{15}$ Service de Biochimie, CHU Hotel Dieu, Nantes and ${ }^{16}$ Département de Recherche Clinique, CHU Hotel Dieu, Nantes, France.
Correspondence:

CYRILLE TOUZEAU - cyrille.touzeau@chu-nantes.fr https://doi.org/10.3324/haematol.2021.280394

Received: December 6, 2021.

Accepted: February 9, 2022.

Prepublished: February 17, 2022.

\section{Disclosures}

$\mathrm{CT}, \mathrm{AP}, \mathrm{LK}, \mathrm{CJ}, \mathrm{MM}, \mathrm{CH}, \mathrm{XL}$ and $\mathrm{PM}$ have received honoraria from Takeda, Amgen, BMS/Celgene and Janssen; MLC has received honoraria from Celgene and Takeda.

\section{Contributions}

The following centers and investigators from the IFM participated in this study: Dijon, Centre Hospitalier du Bocage (D. Caillot, J.N. Bastié, I. Lafon, M.L. Chrétien, E. Ferrant); Lille, Hôpital Claude Huriez (E. Boyle, T. Facon, S. Manier, X. Leleu, C. Herbaux); Nantes, Hôpital Hôtel Dieu (P. Moreau, N. Blin, P. Chevallier, J. Delaunay, V. Dubruille, T. Gastinne, T. Guillaume, S. Le Gouill, B. Mahé, C. Touzeau); Paris, Hôpital St Antoine (M. Mohty, A. Verkhoff); Toulouse, IUC Oncopole (M. Attal, M. Roussel, B. Hebraud); Tours, Hôpital Bretonneau (L. Benbouker, C. Dartigeas, M. Ertault de la Bretonnière, E. Gyan, S. Lissandre, H. Monjanel, M. Renaud, A. Iltis); Lyon, Centre Hopitalier de Lyon Sud (L. KArlin, E. Bachy; F. Bouafia-Sauvy, D. Espinouse, L. Lebras, A-S Michallet, C. Sarkozy, F. Broussais-Guillaumot, B. Coiffier, G. Salles); Bordeaux Hopital Haut Lévêque (C.Hulin, G. Marit, A. Lascaux, S.Dimicoli-Salazar, M. Robles); Nancy, CHU Brabois (A. Perrot, C. Bonmati, D. Ranta, L. Clement-Filiatre, G. Roth Guepin, M. D’Aveni); La Roche Sur Yon CHD les Oudairies (M. Tiab, H. Maisonneuve, B.Villemagne, M. Voldoire); Paris, Hopital St Louis (J.P. Fermand, B. Arnulf, M. Baron, M. Malphettes, B. Asli).

\section{Acknowledgments}

The authors gratefully acknowledge the work performed by individual research teams at all participating study sites; they are also indebted to $\operatorname{Dr}$ C. Mathiot, Dr C. Boccaccio, and L. Biron for their administrative and material support.

\section{Funding}

The study was funded by Takeda Pharmaceuticals.

\section{Data-sharing statement}

The authors will make original data and the study protocol available to other investigators without unreasonable restrictions.

\section{References}

1. Dimopoulos MA, Moreau P, Terpos E, et al. Multiple myeloma: EHA-ESMO clinical practice guidelines for diagnosis, treatment and follow-up. HemaSphere. 2021;5(2):e528.

2. Attal M, Lauwers-Cances $V$, Hulin C, et al. Lenalidomide, bortezomib, and dexamethasone with transplantation for myeloma. N Engl J Med. 2017;376(14):1311-1320.
3. Cavo M, Gay F, Beksac M, et al. Autologous haematopoietic stemcell transplantation versus bortezomib-melphalan-prednisone, with or without bortezomib-lenalidomide-dexamethasone consolidation therapy, and lenalidomide maintenance for newly diagnosed multiple myeloma (EMN02/HO95): a multicentre, randomised, open-label, phase 3 study. Lancet Haematol. 


\section{LETTER TO THE EDITOR}

2020;7(6):e456-e468.

4. Gay F, Musto P, Rota-Scalabrini D, et al. Carfilzomib with cyclophosphamide and dexamethasone or lenalidomide and dexamethasone plus autologous transplantation or carfilzomib plus lenalidomide and dexamethasone, followed by maintenance with carfilzomib plus lenalidomide or lenalidomide alone for patients with newly diagnosed multiple myeloma (FORTE): a randomised, open-label, phase 2 trial. Lancet Oncol. 2021;22(12):1705-1720.

5. Rosiñol L, Oriol A, Rios R, et al. Bortezomib, lenalidomide, and dexamethasone as induction therapy prior to autologous transplant in multiple myeloma. Blood. 2019;134(16):1337-1345.

6. Moreau P, Masszi T, Grzasko N, et al. Oral ixazomib, lenalidomide, and dexamethasone for multiple myeloma. N Engl J Med. 2016;374(17):1621-1634.

7. Kumar S, Paiva B, Anderson KC, et al. International Myeloma Working Group consensus criteria for response and minimal residual disease assessment in multiple myeloma. Lancet Oncol. 2016;17(8):e328-346.

8. Dimopoulos MA, Gay F, Schjesvold F, et al. Oral ixazomib maintenance following autologous stem cell transplantation (TOURMALINE-MM3): a double-blind, randomised, placebocontrolled phase 3 trial. Lancet. 2018;393(10168):253-264.

9. Rosinol L, Oriol A, Ríos Tamayo R, et al. Ixazomib plus lenalidomide/dexamethasone (IRd) versus lenalidomide /dexamethasone ( $\mathrm{Rd}$ ) maintenance after autologous stem cell transplant in patients with newly diagnosed multiple myeloma: results of the Spanish GEM2014MAIN trial. Blood. 2021;138(Suppl 1):466.
10. McCarthy PL, Holstein SA, Petrucci MT, et al. Lenalidomide maintenance after autologous stem-cell transplantation in newly diagnosed multiple myeloma: a meta-analysis. J Clin Oncol. 2017;35(29):3279-3289.

11. Dimopoulos M, Laubach J, Gutierrez M, Richardson PG. Efficacy and safety of long-term ixazomib maintenance therapy in patients (pts) with newly diagnosed multiple myeloma (NDMM) not undergoing transplant: an integrated analysis of four phase 1/2 studies. Blood. 2017;130(Suppl 1):902.

12. Roussel M, Lauwers-Cances V, Robillard N, et al. Frontline therapy with carfilzomib, lenalidomide, and dexamethasone (KRd) induction followed by autologous stem cell transplantation, Krd consolidation and lenalidomide maintenance in newly diagnosed multiple myeloma (NDMM) patients: primary results of the Intergroupe Francophone Du MyéLome (IFM) Krd phase II study. Blood. 2016;128(22):1142.

13. Besse A, Besse L, Kraus M, et al. Proteasome inhibition in multiple myeloma: head-to-head comparison of currently available proteasome inhibitors. Cell Chem Biol. 2019;26(3):340-351.

14. Moreau P, Attal M, Hulin C, et al. Bortezomib, thalidomide, and dexamethasone with or without daratumumab before and after autologous stem-cell transplantation for newly diagnosed multiple myeloma (CASSIOPEIA): a randomised, open-label, phase 3 study. Lancet. 2019;394(10192):29-38.

15. Voorhees PM, Kaufman JL, Laubach JP, et al. Daratumumab, lenalidomide, bortezomib, \& dexamethasone for transplanteligible newly diagnosed multiple myeloma: GRIFFIN. Blood. 2020;136(8):936-945. 